ACCEPTED MANUSCRIPT

\title{
Strategies to tailor the architecture of dual Ag/Fe-oxide nano- heterocrystals - Interfacial and morphology effects on the magnetic behavior
}

To cite this article before publication: Pablo Tancredi et al 2018 J. Phys. D: Appl. Phys. in press https://doi.org/10.1088/1361-6463/aaccc3

\section{Manuscript version: Accepted Manuscript}

Accepted Manuscript is "the version of the article accepted for publication including all changes made as a result of the peer review process, and which may also include the addition to the article by IOP Publishing of a header, an article ID, a cover sheet and/or an 'Accepted Manuscript' watermark, but excluding any other editing, typesetting or other changes made by IOP Publishing and/or its licensors"

This Accepted Manuscript is (C) 2018 IOP Publishing Ltd.

During the embargo period (the 12 month period from the publication of the Version of Record of this article), the Accepted Manuscript is fully protected by copyright and cannot be reused or reposted elsewhere.

As the Version of Record of this article is going to be / has been published on a subscription basis, this Accepted Manuscript is available for reuse under a CC BY-NC-ND 3.0 licence after the 12 month embargo period.

After the embargo period, everyone is permitted to use copy and redistribute this article for non-commercial purposes only, provided that they adhere to all the terms of the licence https://creativecommons.org/licences/by-nc-nd/3.0

Although reasonable endeavours have been taken to obtain all necessary permissions from third parties to include their copyrighted content within this article, their full citation and copyright line may not be present in this Accepted Manuscript version. Before using any content from this article, please refer to the Version of Record on IOPscience once published for full citation and copyright details, as permissions will likely be required. All third party content is fully copyright protected, unless specifically stated otherwise in the figure caption in the Version of Record.

View the article online for updates and enhancements. 


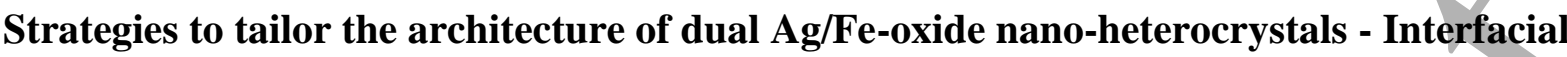
and morphology effects on the magnetic behavior

P. Tancredi ${ }^{1}$, O. Moscoso Londoño ${ }^{2,3 *}$, P.C. Rivas Rojas ${ }^{1}$, U. Wolff ${ }^{4}$, L. M. Socolovsky ${ }^{5}$, M, Knobel $^{2}$, and D. Muraca ${ }^{2}$

${ }^{1}$ Laboratorio de Sólidos Amorfos, Instituto de Tecnologías y Ciencias de la Ingeniería "Hilario Fernández Long”, Facultad de Ingeniería, Universidad de Buenos Aires - CONICET, Buenos Aires, Argentina

${ }^{2}$ Laboratório de Materiais e Baixas Temperaturas, Instituto de Fïsica 'Gleb Wataghin', Universidade Estadual de Campinas, Campinas, Brazil

${ }^{3}$ Facultad de Ingeniería, Universidad Autónoma de Manizales, Antigua Estación del Ferrocarril, Manizales, Colombia.

${ }^{4}$ IFW Dresden, Leibniz Institute for Solid State and Materials Research, Dresden, Helmholtzstrasse 20, 01069 Dresden, Germany

${ }^{5}$ Facultad Regional Santa Cruz, Universidad Tẹcnológica Nacional - CIT Santa Cruz (CONICET), Río Gallegos, Argentina

*Corresponding author: omoscoso@ifi.unicamp.br, oscar.moscosol@autonoma.edu.co 
Abstract

Bifunctional nanostructured architectures have shown appealing properties, since a single entity can combine the diverse properties of its individual constituents. Particularly, by growing Feoxide domains over Ag nanoparticles, the plasmonic and superparamagnetic properties can be combined in a single particle. Beyond the multifunctionality of this system, there are several properties that emerge from intrinsic factors, such as: interface and/or morphology. In this study, we present the synthesis protocols to obtain two sets of heterocrystals, each one with different morphology: dimer and flower-like. In addition, the magnetization behavior of these hybrid nano-heterocrystals is investigated and discussed. These nanomaterials were built by a seed assisted heterogeneous nucleation process, carried out in organic solvents of high boiling point, using the same batch of silver nanoparticles with a mean size of $6 \mathrm{~nm}$ as seeds, and tuning the electron-donor capacity of the reaction environment at the thermal decomposition of the iron precursor. $\mathrm{Ag} / \mathrm{Fe}_{3} \mathrm{O}_{4}$ heterocrystals with dimer and flower-like morphologies were obtained. The synthesis protocols for generating these types of nanomaterials are discussed step-by-step. Structural and morphological properties were determined by transmission electron microscopy (TEM), X-ray diffraction (XRD) and X-ray absorption fine structure (XAFS). DC magnetization results suggest that the silver/magnetite coupling generates an increase of the blocking temperature in comparison to those obtained from pure magnetite. This behavior could be linked to a possible increase in the magnetic anisotropy produced by an additional disorder at the Ag$\mathrm{Fe}_{3} \mathrm{O}_{4}$ interface. The higher interface area of the $\mathrm{Ag} / \mathrm{Fe}_{3} \mathrm{O}_{4}$ heterocrystals with flower-like architecture leads to a higher blocking temperature and a stronger magnetic anisotropy. These results are supported by $\mathrm{AC}$ susceptibility data.

Keywords: hybrid nanostructures, interface effects, magnetic-plasmonic properties, superparamagnetism. 


\section{Introduction}

In the last years, a prominent part of the research in nanochemistry has focused on the preparation of multifunctional and heterostructured nanocrystals, capable of combining two or more inorganic materials into a single system through a direct atomic bond at the interface and without the presence of a molecular bridge [1-3]. In these new heterocrystals, the properties of the different components can be combined or can give rise to new features associated to morphology or interface effects, factors that may partially govern the physicochemical behavior of these new kind of systems.

Nanoparticles that combine a noble metal ( $\mathrm{Au}, \mathrm{Ag})$ with ferrite type oxides $\left(\mathrm{Fe}_{3} \mathrm{O}_{4}, \mathrm{CoFe}_{2} \mathrm{O}_{4}\right.$, $\left.\mathrm{Co}_{3} \mathrm{O}_{4}, \mathrm{MnFe}_{2} \mathrm{O}_{4}\right)$ are among the systems that have gained major interest. In these peculiar nanostructures, the optical properties of metallic nanoparticles and the magnetic properties of Feoxide counterparts can be coupled. This dual behavior opens the door to new applications in several fields, including catalysis [4, 5], antimicrobial agents [6, 7], magnetic and photo/magnetic hyperthermia therapies [8, 9] and surface-enhanced Raman scattering substrates (SERS) [10], among others.

The most common process to produce heterostructured nanocrystals is the so-called seed-assisted method, where a second component is heterogeneously nucleated over previously formed nanoparticles ("seeds") of a first component. In this process, the heterogeneous nucleation of the second material is favored over homogeneous nucleation, especially when there is some compatibility between the cell parameters of the crystals [10]. Depending on the nucleation events and the growth evolution, the formed heterostructures can show distinct architectures, such as core-shell, dimer or flower-like. Many efforts have been dedicated to the understanding of the synthesis conditions and mechanisms that lead to these different morphologies [2, 11-13], mainly because the macroscopic properties of the system can be controlled or tuned by the interface type and the interactions between the different constituents.

In this work, we study the synthesis and the magnetic response of two $\mathrm{Ag} / \mathrm{Fe}_{3} \mathrm{O}_{4}$ heterostructures with different morphologies: dimer and flower-like type, prepared by a seed-assisted route in 
organic solvents of high boiling point. In particular, we investigate how the solvent nature, associated to its electron-donor capacity, determines the final morphology. Correspondingly, we performed a complete magnetic characterization by means of DC and AC magnetization techniques to understand how the heterostructures architecture and the distribution of the $\mathrm{Fe}_{3} \mathrm{O}_{4}$ domains define the macroscopic behavior of the samples. A discussion on the structural and optical properties is also presented to reinforce the results and the conclusions.

\section{Experimental Details}

Materials. Silver nitrate $\left(\mathrm{AgNO}_{3}, \geq 99 \%\right.$, Sigma-Aldrich), sodium acetate $\left(\mathrm{NaCH}_{3} \mathrm{COO}, \geq 99 \%\right.$, Anedra), oleylamine $\left(\mathrm{C}_{17} \mathrm{H}_{31}-\mathrm{CH}_{2} \mathrm{NH}_{2}, \geq 99 \%\right.$, Sigma-Aldrich), oleic acid $\left(\mathrm{C}_{17} \mathrm{H}_{31}-\mathrm{COOH}, 90 \%\right.$, Sigma-Aldrich), 1-octadecene $\left(\mathrm{C}_{18} \mathrm{H}_{36}, 90 \%\right.$, Sigma-Aldrich), diphenyl ether $\left(\left(\mathrm{C}_{6} \mathrm{H}_{5}\right)_{2} \mathrm{O}, 98 \%\right.$, Sigma-Aldrich), iron(III) acetylacetonate (Fe(acac) $3, \quad 99 \%, \quad$ Sigma-Aldrich) and 1,2hexadecanediol $\left(\mathrm{C}_{16} \mathrm{H}_{34} \mathrm{O}_{2}, 90 \%\right.$, Sigma-Aldrich). All chemicals were used as received without further purification.

Methods. $\mathrm{Ag} / \mathrm{Fe}_{3} \mathrm{O}_{4}$ nanoparticles were synthesized by a seed assisted heterogeneous nucleation process in organic solvents of high boiling point, as described below.

Silver Seeds Nanoparticles (AgNPs) synthesis: AgNPs were prepared according to previous reports [8, 12]. Briefly, $2 \mathrm{mmol}$ silver acetate $\left(\mathrm{AgCH}_{3} \mathrm{COO}\right)$ were obtained by stochiometric precipitation of $\mathrm{AgNO}_{3}$ and $\mathrm{NaCH}_{3} \mathrm{COO}$. The so-obtained white powder was dissolved in 2 mmol oleic acid, 2 mmol oleylamine and $5 \mathrm{~mL}$ 1-octadecene. The mixture was heated up to 175 ${ }^{\circ} \mathrm{C}$ under continuous stirring for $30 \mathrm{~min}$ to allow AgNPs formation. Finally, the system was cooled down to room temperature and the AgNPs were precipitated by the addition of ethanol and isolated by centrifugation at 3000 RPM for 15 minutes. The isolated solid was washed 3 times before redispersed in hexane.

$\mathrm{Ag} / \mathrm{Fe}_{3} \mathrm{O}_{4}$ heteroparticles formation: AgNPs were used as fixed seeds for $\mathrm{Fe}_{3} \mathrm{O}_{4}$ heterogeneous nucleation. $1 \mathrm{mmol} \mathrm{Ag} \mathrm{(as} \mathrm{AgNPs),} 0.3 \mathrm{mmol} \mathrm{Fe}(\mathrm{acac})_{3}, 1.5 \mathrm{mmol}$ 1,2-hexadecanediol, 0.75 mmol oleic acid, $1.5 \mathrm{mmol}$ oleylamine and $5 \mathrm{~mL}$ solvent (1-octadecene, ODE or phenyl ether, 


\section{Characterization}

Phase composition and crystallographic characterization were carried out via X-ray diffraction (XRD) on a standard Rigaku diffractometer with $\mathrm{Cu} \mathrm{K}-\alpha$ radiation. X-ray absorption fine structure (XAFS) spectroscopy patterns for the Fe K-edge $(7.112 \mathrm{keV})$ were recorded at room temperature in fluorescence mode with the colloidal samples positioned at $45^{\circ}$ with respect to the X-ray beam. These measurements were performed at the Brazilian Synchrotron Light Laboratory (CNPEM/LNLS, Campinas, Brazil). Absorption spectra, carried out on colloidal samples (nanoparticles dispersed in toluene), were recorded at room temperature by means of a UV-VisNIR Shimadzu UV-1603 using quartz cuvettes with $10 \mathrm{~mm}$ optical path length. Morphology and size distribution of the samples were determined by transmission electron microscopy (TEM) at the CNPEM/LNNano (Campinas, Brazil) in a JEOL JEM 2100F with a field emission gun (FEG) operating at $200 \mathrm{kV}$ and at the IFW (Dresden, Germany) with a FEI-Tecnai microscope operating at $200 \mathrm{kV}$. The samples for TEM experiments were prepared by dripping $10 \mu \mathrm{L}$ of nanoparticle dispersion onto a carbon-coated copper grid (Ted Pella) and drying the solvent at room temperature. DC magnetization and AC magnetic susceptibility measurements were recorded with a MPMS-XL7 Quantum Design SQUID magnetometer and a PPMS Quantum Design magnetometer, respectively. The samples for magnetic measurements were prepared by mixing $50 \mu \mathrm{L}$ of the colloidal dispersion containing the $\mathrm{Ag} / \mathrm{Fe}_{3} \mathrm{O}_{4}$ heterocrystals and one drop of 1-octadecene. This mixture was stirred until the toluene was evaporated. After that, a piece of 
cotton (appr $15 \mathrm{mg}$ ) was impregnated with the $\mathrm{Ag} / \mathrm{Fe}_{3} \mathrm{O}_{4}$ sample and finally placed in the SQUID/PPMS holder sample.

\section{Results and Discussion}

The two-step procedure employed for the $\mathrm{Ag} / \mathrm{Fe}_{3} \mathrm{O}_{4}$ heteroparticles synthésis can be conveniently used to study the reaction mechanism and the conditions that lead to the formation of different heterostructure morphologies.

The AgNPs (produced separately in a first step) were incorporated to the synthesis medium prior to the thermal decomposition reaction and the formation of the iron oxide phase. One-step reactions have been successfully developed to produce heterocrystals by exploiting the difference between the formation temperatures of the metallic nanoparticle and the iron oxide [14-16]. In spite of this, we have decided to employ a two-step procedure so we can directly relate the heterogeneous nucleation events and final heteroparticle structure with the reaction conditions, i.e. solvent electron-donor capacity, and detach them from other variables, such as AgNPs size and concentration, for example. Reports on two-steps heterostructure synthesis have investigated different options for the iron source, including iron acetylacetonate, iron oleate or iron pentacarbonyl. However, the precursor nature does not seem to affect the final particle conformation because a common transformation to iron oleate occurs at low temperatures (prior to iron oxide nucleation) regardless the original reagent $[17,18]$.

Fig. 1 shows a representative TEM image of the prepared AgNPs. The synthesized AgNPs show a spheroidal-like shape with a narrow size distribution. Histograms were obtained by counting more than 200 silver nanoparticles from several TEM images, which were well fitted using a lognormal function (Fig. 1(b)). From this, a mean diameter $\langle D\rangle=6 \mathrm{~nm}$ with a rather narrow size distribution $(\sigma=0.2)$ was determined. These results confirm the well-dispersed nature of the initial AgNPs colloidal suspension obtained in the first step of the synthesis. The $f c c$ structure of the silver nanocrystals was examined by X-ray diffraction, as it is shown in Fig. S1 (Supplementary Information). 

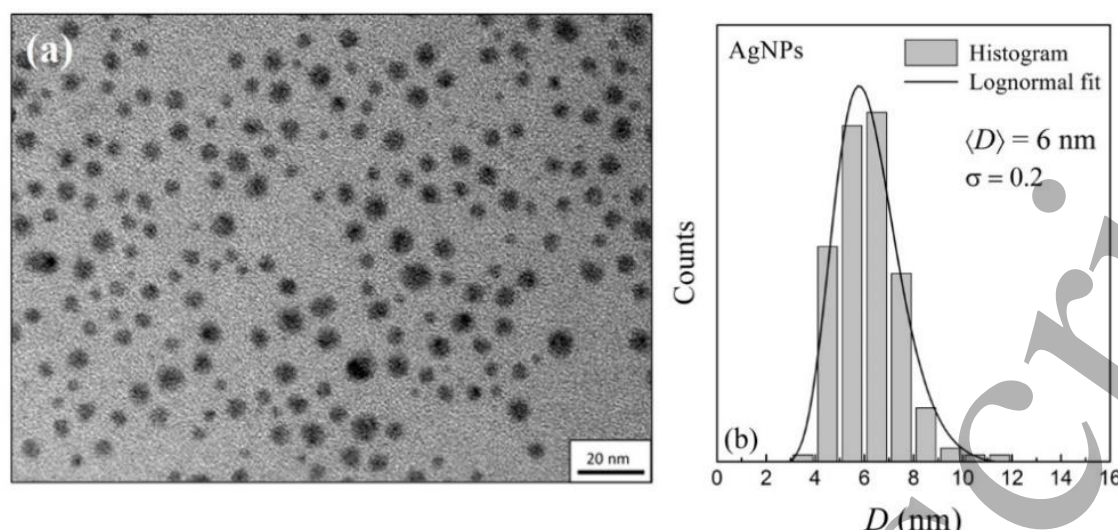

$D(\mathrm{~nm})$

Figure 1. (a) TEM micrograph of AgNPs. (b) Size distribution histogram and the lognormal fit.

Afterwards, these AgNPs were used as fixed seeds for the formation of the $\mathrm{Ag} / \mathrm{Fe}_{3} \mathrm{O}_{4}$ heteroparticles in both reaction solvents. Fig. 2 and 3 show the TEM and HR-TEM of the two samples. The difference in the atomic number between the two components makes it possible to distinguish iron oxide (lighter particles) from $\mathrm{Ag}$ (darker particles) in the images. For the heterostructures prepared in 1-octadecene $\left(\mathrm{Ag} / \mathrm{Fe}_{3} \mathrm{O}_{4} \mathrm{ODE}\right)$ dimer-like heteroparticles are predominant (Fig. 2), with just one iron oxide crystal attached per Ag seed. On the other hand, for heterostructures prepared in phenyl ether $\left(\mathrm{Ag} / \mathrm{Fe}_{3} \mathrm{O}_{4} \mathrm{PHE}\right)$ flower-like heteroparticles predominate (Fig. 3), with various iron oxide crystals surrounding a central Ag seed. In both TEM images (Fig. 2(a) and Fig. 3(a)), some non-attached iron oxide particles can be seen. However, the presence of these particles decreased dramatically from previous samples by carefully tuning the relation between $\mathrm{Ag}$ seeds and Fe precursor and the heating rate. Fig. 2(d) and 3(d) present the HR-TEM images for samples $\mathrm{Ag} / \mathrm{Fe}_{3} \mathrm{O}_{4} \mathrm{ODE}$ and $\mathrm{Ag} / \mathrm{Fe}_{3} \mathrm{O}_{4} \mathrm{PHE}$, respectively. Additional images are displayed in the Supporting Information (Fig. S2 and S3). Notice that by applying the Fourier transformation technique on a simple dimer-like particle (inset on Fig. 2(d)), characteristic crystallographic planes for magnetite and silver phases can be detected, i.e., (2 2 2) plane for the $\mathrm{Fe}_{3} \mathrm{O}_{4}$ and (2 0 0) plane for the Ag. For the sample $\mathrm{Ag} / \mathrm{Fe}_{3} \mathrm{O}_{4}$ PHE, the Fourier transformation (inset on Fig. 3(d)) reveals two characteristic crystallographic planes for magnetite phase: (2 200 ) and ( $\left.\begin{array}{lll}3 & 1 & 1\end{array}\right)$. However, none of the crystallographic planes of the metallic silver are distinguished, which is probably related to the absence of the oriented planes of this material in the obtained HR-TEM image. 

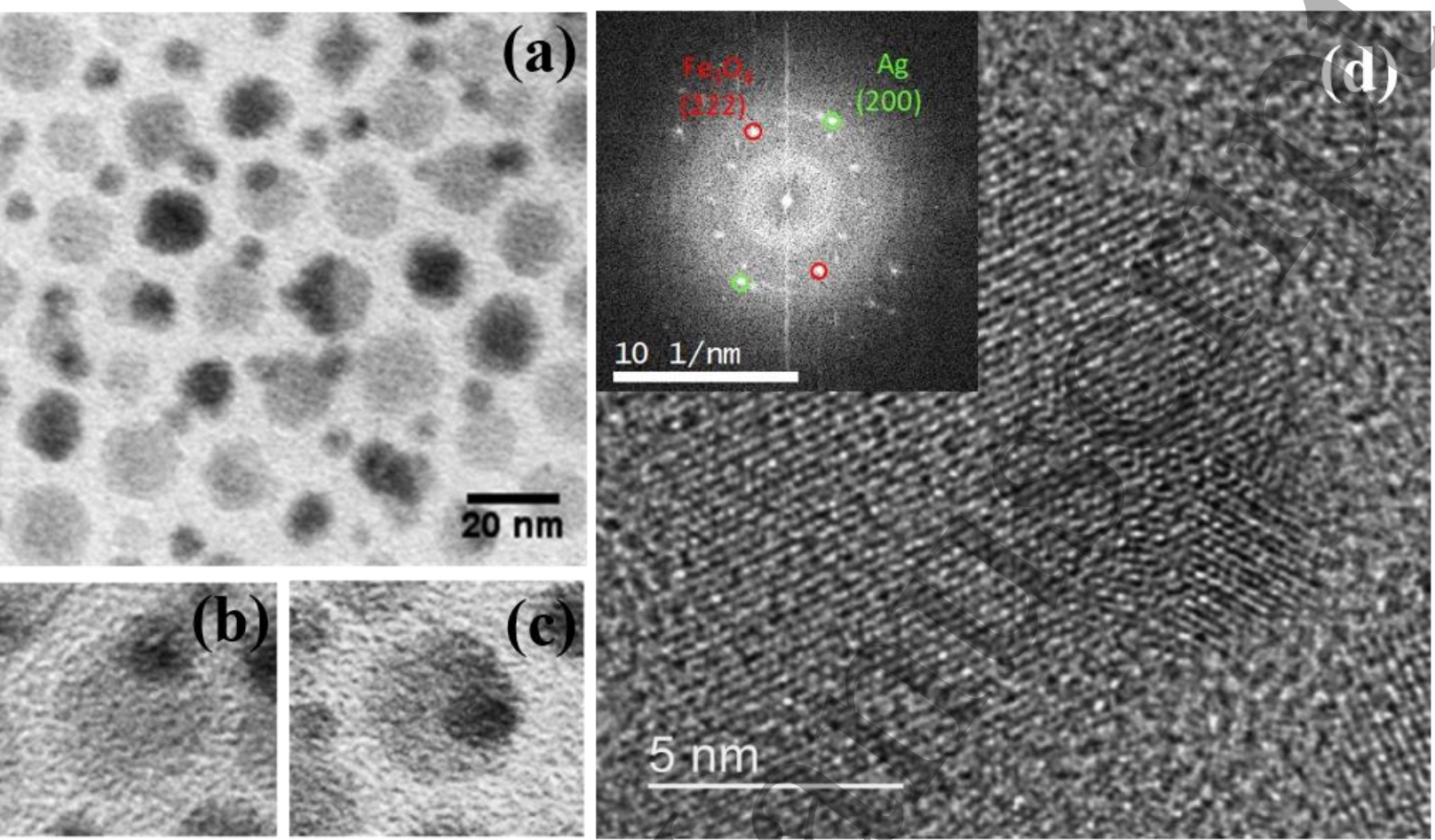

Figure 2. (a-c) Representative TEM images of $\mathrm{Ag} / \mathrm{Fe}_{3} \mathrm{O}_{4}$ heterostructures with dimer-like configuration showing the iron oxide nanoparticles attached to a silver seed. (d) HR-TEM image of a single dimer-like nanoparticle and corresponding Fourier transform (inset). 

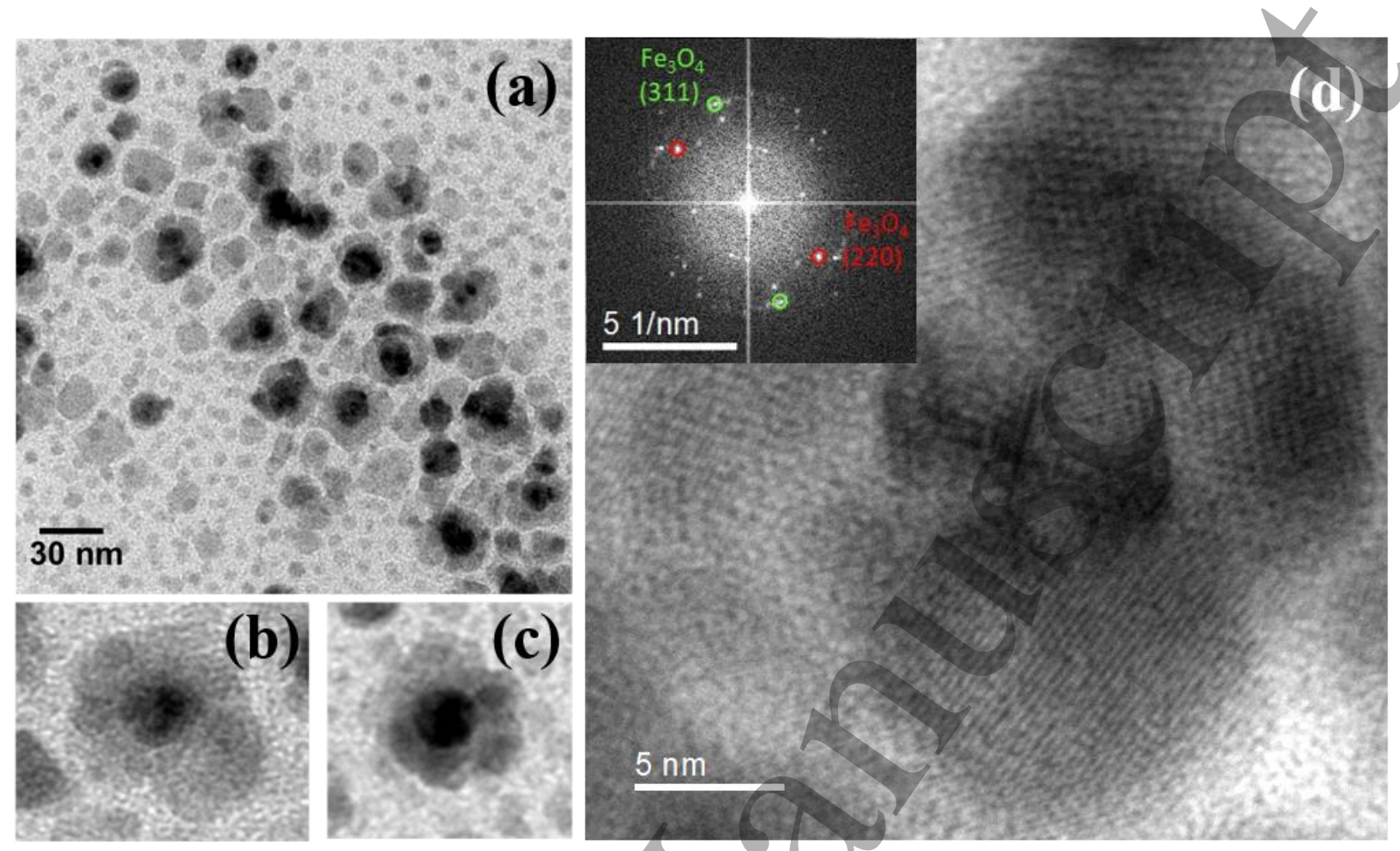

Figure 3. (a-c) Representative TEM images of $\mathrm{Ag} / \mathrm{Fe}_{3} \mathrm{O}_{4}$ heterostructures with flower-like configuration showing the iron oxide nanoparticles surrounding a silver seed. (d) HR-TEM image of a single flower-like nanoparticle. (d) HR-TEM image of a single dimer-like nanoparticle and corresponding Fourier transform (inset).

Fig. 4(a) shows the XRD patterns of the dried heterostructures. As expected, two contributions from two sets of diffraction peaks can be observed in the diffractograms. One set can be assigned to the $f c c$ spinel-inverse iron oxide phase $\left(\mathrm{Fe}_{3} \mathrm{O}_{4}\right.$ and/or $\left.\gamma-\mathrm{Fe}_{2} \mathrm{O}_{3}\right)$ and the other one corresponds to $f c c$ metallic Ag. Thus, one can confirm the presence of both crystalline structures in the samples.

Optical properties were studied in terms of UV-Visible absorption and the resulting spectra are shown in Fig. 4(b). The surface plasmon resonance peak associated to the Ag seeds in the heterocrystals is weakened compared to that obtained from the measurement of pure AgNPs. This behavior is due to the shielding effect caused by $\mathrm{Fe}_{3} \mathrm{O}_{4}[19,20]$. Comparing both samples, the effect is more pronounced for $\mathrm{Ag} / \mathrm{Fe}_{3} \mathrm{O}_{4} \mathrm{PHE}$ than for $\mathrm{Ag} / \mathrm{Fe}_{3} \mathrm{O}_{4} \mathrm{ODE}$. This difference can be related to the morphology dissimilarities, as flower-like structures with a larger $\mathrm{Ag}-\mathrm{Fe}_{3} \mathrm{O}_{4}$ interface can produce a larger shielding effect compared to dimer-like particles. A similar decay 
of the absorption peak was also observed in $\mathrm{Au} / \mathrm{Fe}_{3} \mathrm{O}_{4}$ particles $[19,21]$. A control sample of 10 $\mathrm{nm}$ magnetite particles was also investigated for comparison (synthesis and characterization details are presented in the Supplementary Information), the respective UV-Vis spectra (red line in Fig. 4(b)) is consistent with subwavelength sized dielectric spheres [22].

In most reports concerning the studies of surface plasmon resonance on metallic/magnetic hybrid nanoparticles, including $\mathrm{Au} / \mathrm{Fe}_{3} \mathrm{O}_{4}$ and $\mathrm{Ag} / \mathrm{Fe}_{3} \mathrm{O}_{4}$, a red-shift of the metal particle surface plasmon resonance peak (SRP) is evidenced. According to some authors, this behavior results due to the strong coupling of iron oxide, which exhibits higher values of the real part of the refractive index with respect to the organic solvents [23, 24]. Strikingly, the absorption peak of our prepared $\mathrm{Ag} / \mathrm{Fe}_{3} \mathrm{O}_{4}$ heteroparticles stays at almost the same position as that of AgNPs. This behavior was also observed by Huang et. al. [20] and can indicate a weak interaction between the building blocks. Some authors also suggest that $\mathrm{Fe}_{3} \mathrm{O}_{4}$ growth over the $\mathrm{Ag}$ seed does not always occur epitaxially, leading to nearly independent surface plasmonic properties of the metallic nanoparticles [19]. Also, some kind of centrifugation sorting during $\mathrm{Ag} / \mathrm{Fe}_{3} \mathrm{O}_{4}$ washing steps may have privileged AgNPs with a UV-shifted peak position. Moreover, since the modification of the size of the scattering objects are known to affect the intensity and width of the SPR peak, the observed differences could also be related to the minor changes in the Ag seeds size during the second synthesis step.

Measurements of X-ray absorption fine structure (XAFS) were carried out in order to examine the Fe-oxidation states of the iron oxide domains in the heterostructures. A control sample of $\mathrm{Fe}_{3} \mathrm{O}_{4}$ nanoparticles was also analyzed for comparison. Fig. 4(c) and (d) show the profiles of the X-ray absorption near edge spectra (XANES) and extended X-ray absorption fine structure (EXAFS), respectively, determined for the samples $\mathrm{Ag} / \mathrm{Fe}_{3} \mathrm{O}_{4} \mathrm{ODE}$ and $\mathrm{Ag} / \mathrm{Fe}_{3} \mathrm{O}_{4} \mathrm{PHE}$, as well as for the iron oxide control sample. According to these results, the pre-edge energy position, the pre-peak features and the EXAFS profiles of both heteroparticles systems are compatible with a structure that combines ferric $\left(\mathrm{Fe}^{2+}\right)$ and ferrous $\left(\mathrm{Fe}^{3+}\right)$ ions [14]. Furthermore, by comparing the obtained results from the $\mathrm{Ag} / \mathrm{Fe}_{3} \mathrm{O}_{4}$ with those one from the $\mathrm{Fe}_{3} \mathrm{O}_{4}$ control sample, one can affirm that the environment of Fe-atoms located in the heterostructures are disposed to form an iron oxide with a stoichiometric $\mathrm{Fe}_{3} \mathrm{O}_{4}$ phase. 

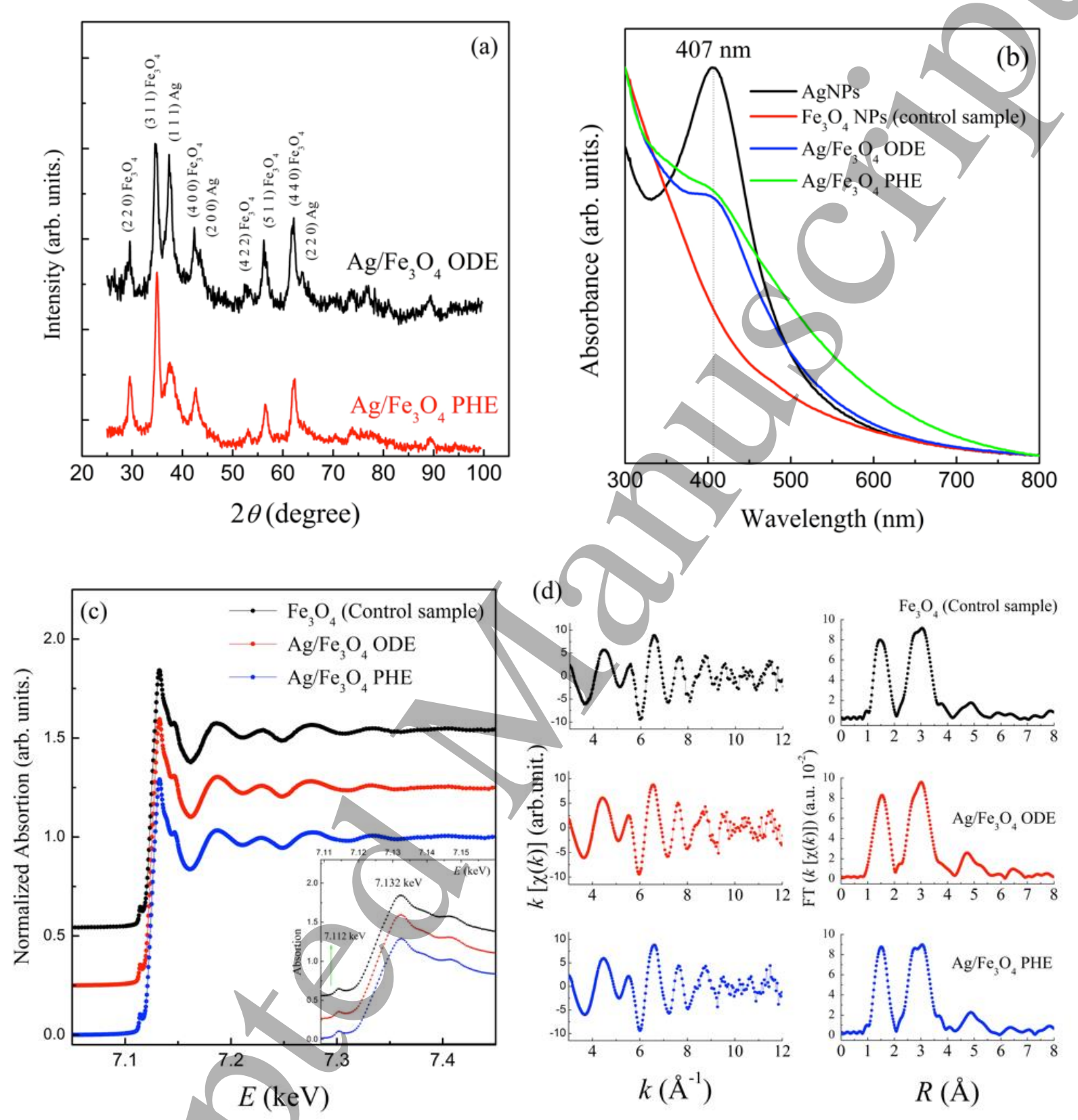

Figure 4. (a) XRD patterns of sample $\mathrm{Ag} / \mathrm{Fe}_{3} \mathrm{O}_{4} \mathrm{ODE}$ (dimer-like morphology) and $\mathrm{Ag} / \mathrm{Fe}_{3} \mathrm{O}_{4}$ PHE (flower-like morphology). The phase and Miller index of principal peaks are indicated. (b) UV-Vis absorption spectra of the samples. (c) Experimental EXAFS Fe K-edge spectra for samples $\mathrm{Ag} / \mathrm{Fe}_{3} \mathrm{O}_{4}$ ODE, $\mathrm{Ag} / \mathrm{Fe}_{3} \mathrm{O}_{4}$ PHE and $\mathrm{Fe}_{3} \mathrm{O}_{4}$. Inset: XANES region. (d) Fourier transformations obtained for the studied samples. 
The study and control of the reaction conditions leading to different heterostructures morphologies has been a topic of intense research in the last years. In a pioneering work, $\mathrm{H}$. $\mathrm{Yu}$ and co-workers proposed that a charge compensation process within the metal nanoparticle (Au in their case) governs and defines the heterostructure conformation [11]. The nucleation of the iron oxide on the $\mathrm{Au}$ surface induces a positive charge at the growth interface, immediately compensated by free electrons of the metal. This charge migration produces electron deficient "restrained facets" and denies multi-nucleation process. However, a change in the medium's electron-donor capacity by using a slightly polar solvent could compensate the charge induced by the polarized growth plane, and thus allow multi-nucleation. Therefore, the modification of the reaction medium from octadecene to phenyl ether can modify the final heterostructure conformation from dimer-like to flower-like nanoparticles. Fig. 5 shows a scheme of the possible pathways for the seeded-growth synthesis of $\mathrm{Ag} / \mathrm{Fe}_{3} \mathrm{O}_{4}$ heterocrystals starting from a mixture of $\mathrm{Ag}$ seeds and Fe precursor. This hypothesís was supported by few authors for noble metal (especially $\mathrm{Au}$ ) heterostructures $[11,12,20,25]$, and also for other metal - metal oxide combinations, such as FePt/MnO [26]. Flower-like nanoparticles were also produced in highly polar solvents like poly-alcohols [10], supporting the hypothesis that the polarity of the synthesis medium influences the morphology of the heterostructure. Nevertheless, additional reports suggest that other reaction conditions like metal seeds - Fe precursor ratio [27] or metal seed crystallinity [20] could also define the heterostructure configuration. 
(a)

Heterogeneous
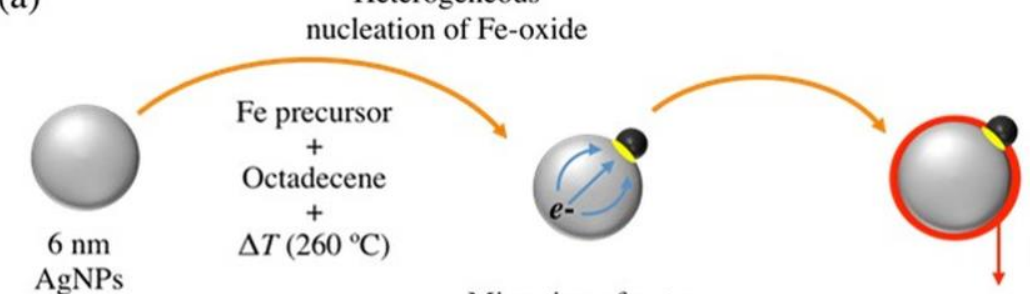

Migration of e- to compensate the charge at the interface

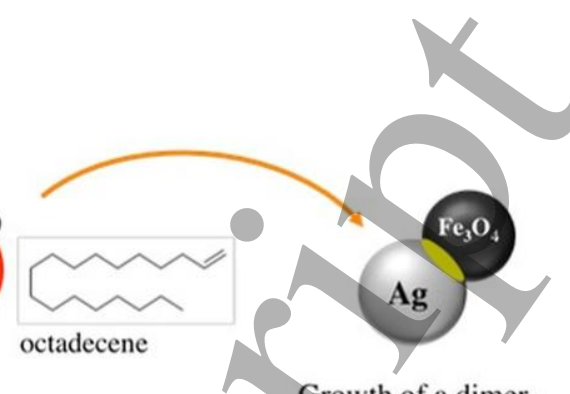

Growth of a dimerlike structure

(b)

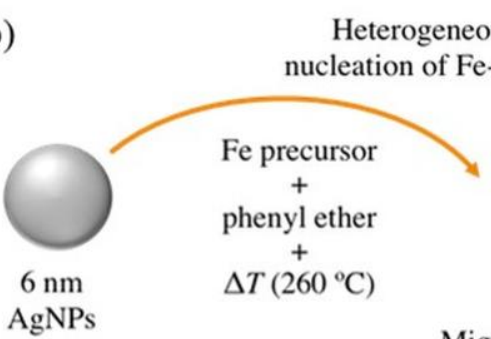

Migration of e- to compensate the charge at the interface

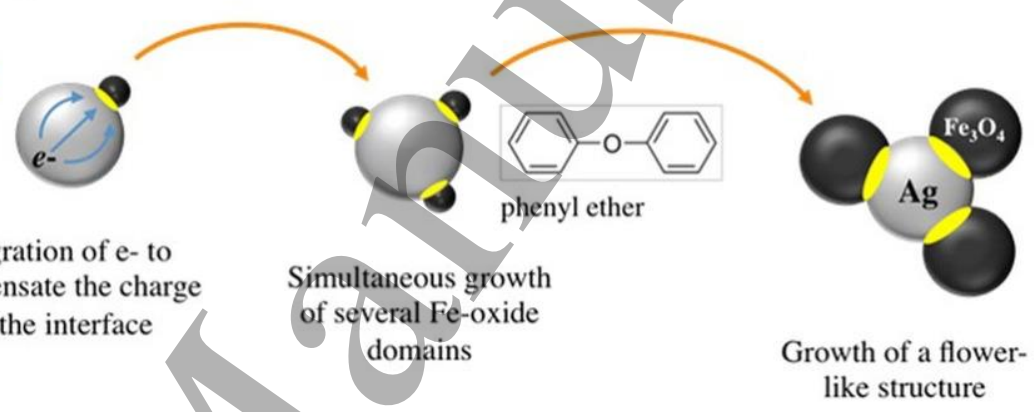
for nucleation like structure

Figure 5. Schematic representation of the two possible pathways for the seeded-growth formation of $\mathrm{Ag} / \mathrm{Fe}_{3} \mathrm{O}_{4}$ heterocrystals with different morphology. Heterogeneous nucleation of $\mathrm{Fe}_{3} \mathrm{O}_{4}$ to form (a) dimer or (b) flower-like structure.

Magnetization features of both $\mathrm{Ag} / \mathrm{Fe}_{3} \mathrm{O}_{4}$ systems were investigated in order to understand the influence of the nanoparticle morphology on the macroscopic magnetic behavior. Because the magnetic measurements were performed in highly diluted samples, interparticle dipolar interactions are expected to be negligible. However, on the sample with flower-like morphology, the presence of several $\mathrm{Fe}_{3} \mathrm{O}_{4}$ domains attached to a single $\mathrm{Ag}$ seed can lead to intra particle dipolar and/or exchange interactions, because these magnetic domains can be in direct contact with each other and not spatially separated by the diamagnetic matrix.

Fig. 6 shows the magnetization vs. applied field ( $M$ vs. $H$ ) curves performed at $5 \mathrm{~K}$ and $100 \mathrm{~K}$. Notice that the magnetization units are expressed in terms of emu per mass of heteroparticles, $i$. e., mass of $\mathrm{Fe}_{3} \mathrm{O}_{4}$ and $\mathrm{Ag}$. While the saturation magnetization $\left(M_{\mathrm{S}}\right)$ values are lower than those reported for bulk magnetite, as expected for nanoscale magnetic materials, these are partially 
underestimated because the additional mass of silver. The $S$-shaped curves can be related to the presence of monodomain magnetic iron oxide nanoparticles. At $T=5 \mathrm{~K}$ the magnetization loops display a coercive field of $\sim 260 \mathrm{Oe}$ for the $\mathrm{Ag} / \mathrm{Fe}_{3} \mathrm{O}_{4} \mathrm{ODE}$ sample and $\sim 360$ Oe for the $\mathrm{Ag} / \mathrm{Fe}_{3} \mathrm{O}_{4}$ PHE sample, indicating that the nanoparticles are at the magnetically blocked regime at this temperature [28]. As can be noted, the coercive field at $5 \mathrm{~K}$ is larger in the $\mathrm{Ag} / \mathrm{Fe}_{3} \mathrm{O}_{4} \mathrm{PHE}$ sample than in the $\mathrm{Ag} / \mathrm{Fe}_{3} \mathrm{O}_{4} \mathrm{ODE}$ sample. This behavior can be understood from two main approaches: $(i)$ Because the presence of several iron oxide domains attached to the same AgNP in the sample with a flower-like architecture promotes a strengthening of dipole-dipole interactions between the magnetic entities [12], which could produce a magnetic hardening in this sample. (ii) Because the effective coupling between the components can alter the arrangement of those magnetic moments located at the magnetic surface and thus modifies the magnetization dynamics. The coercive field in both samples drastically diminishes at $100 \mathrm{~K}$, an indication that the magnetization behavior is close to the superparamagnetic regime [28].
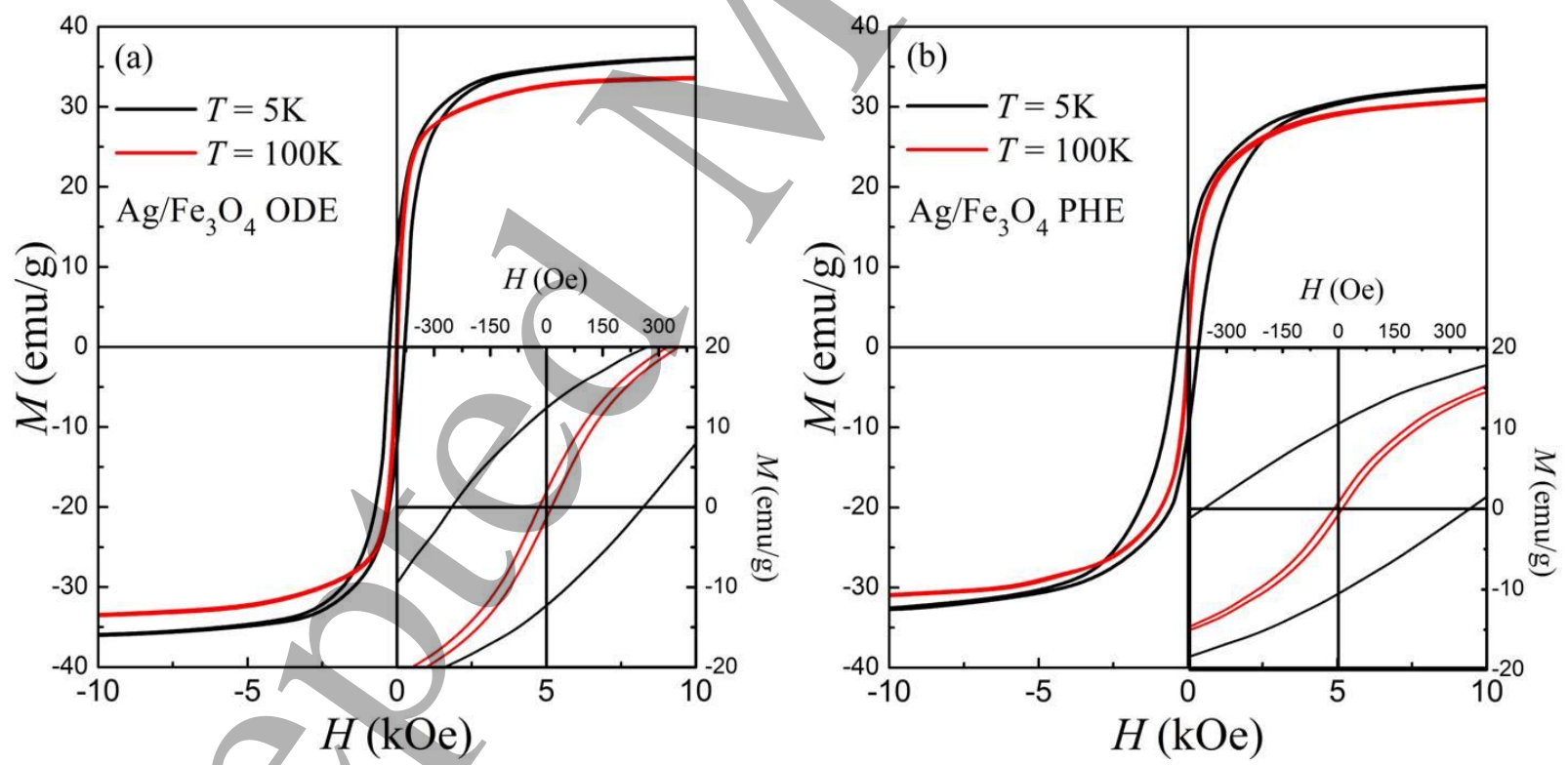

Figure. 6. Magnetization vs. applied magnetic field curves measured at $5 \mathrm{~K}$ and $100 \mathrm{~K}$ for samples (a) $\mathrm{Ag} / \mathrm{Fe}_{3} \mathrm{O}_{4} \mathrm{ODE}$ and (b) $\mathrm{Ag} / \mathrm{Fe}_{3} \mathrm{O}_{4}$ PHE. Insets: zoom-in of the low field region showing the coercivity behavior. 
Temperature dependence of the magnetization of $\mathrm{Ag} / \mathrm{Fe}_{3} \mathrm{O}_{4}$ heterostructures, recorded under zero-field-cooling $\left(M_{\mathrm{ZFC}}\right)$ and field-cooling $\left(M_{\mathrm{FC}}\right)$ protocols, is presented in Fig. 7. In both curves, the ZFC magnetization display a clear and broad maximum value, at $T_{\mathrm{m}}=117 \mathrm{~K}$ for $\mathrm{Ag} / \mathrm{Fe}_{3} \mathrm{O}_{4} \mathrm{ODE}$ and at $T_{\mathrm{m}}=197 \mathrm{~K}$ for $\mathrm{Ag} / \mathrm{Fe}_{3} \mathrm{O}_{4} \mathrm{PHE}$. Although in principle, $T_{\mathrm{m}}$ can be associated to the blocked-to-superparamagnetic transition of the iron oxide domains, the obtained values are approximately one magnitude of order larger than ones obtained for the magnetite control sample, which was found at $T_{\mathrm{m}}=27 \mathrm{~K}$ (blue symbols in Fig. 7(a)) and whose shape can be easily linked to a weakly interacting monodomain system [28, 29]. The differences of $T_{\mathrm{m}}$ between the control sample and the more complex nanostructures can be explained considering three different phenomena: changes in the size of the magnetic nanoparticles [30], strengthening of dipolar interactions [31] and variations in the iron oxide phases [32]. However, for both heterostructures the sizes of the magnetic domains are quite similar; hence the magnetic phase remains unaltered and the dipole-dipole and superexchange interactions should not strong enough to generate a shift of $T_{\mathrm{m}}$ of $90 \mathrm{~K}$ and $170 \mathrm{~K}$ in the $\mathrm{Ag} / \mathrm{Fe}_{3} \mathrm{O}_{4}$ ODE and $\mathrm{Ag} / \mathrm{Fe}_{3} \mathrm{O}_{4} \mathrm{PHE}$ samples, respectively (in comparison to the control $\mathrm{Fe}_{3} \mathrm{O}_{4}$ sample). Such interactions, especially in the sample with flower-like morphology, could affect the effective anisotropy of the structures, leading to a $T_{\mathrm{m}}$ displacement (as discussed previously) but in a weaker degree. Generally speaking, the $T_{\mathrm{m}}$ shift (as well as the ZFC/FC general curve shape) can be influenced by the morphology, metal seeds features and/or interface effects. For instance, some authors suggest that the coupling efficiency between metal/Fe-oxide surfaces can modify the superficial disorder [33, 34], the energy barrier $\left(E_{\mathrm{b}}\right)$ and the magnetocrystalline anisotropy $(K)$ of the magnetic counterpart [35], altering the global magnetic behavior of the system. Therefore, two ZFC curves, with values for $T_{\mathrm{m}}$ matching those obtained from the experimental measurements of the $\mathrm{Ag} / \mathrm{Fe}_{3} \mathrm{O}_{4}$ heteroparticles were simulated following the expression reported in [36], which is:

$$
M_{\mathrm{ZFC}}(T) \cong \frac{M_{\mathrm{S}}^{2} H}{3 K}\left[\frac{E_{\mathrm{bm}}}{k_{\mathrm{B}}} \int_{0}^{T / T_{\mathrm{bm}}} \frac{1}{T} y f(y) d y+\int_{T / T_{\mathrm{bm}}}^{\infty} f(y) d y\right],
$$

where $f(y)$ is a distribution of energy barriers (adopted as lognormal-type), $y=E_{\mathrm{b}} / E_{\mathrm{bm}}$ being $E_{\mathrm{b}}$ and $E_{\mathrm{bm}}$ the energy barrier and the mean energy barrier, respectively. $T_{\mathrm{bm}}$ is the mean blocking temperature, $M_{\mathrm{S}}$ the saturation magnetization, $H$ the external magnetic field (50 Oe), $K$ the anisotropy and $k_{\mathrm{B}}$ the Boltzmann constant. These simulations allow us to approximately 
quantify the variations on $E_{\mathrm{bm}}$ and $K_{e f}$ suggested by the mentioned factors. As expected, the $E_{\mathrm{bm}}$ values extracted from the simulated curves (blue dotted lines in Fig. 7) are larger in comparison to the one obtained from the ZFC fit of the $\mathrm{Fe}_{3} \mathrm{O}_{4}$ control sample: $\sim 3 \times 10^{-13}$ erg for $\mathrm{Ag} / \mathrm{Fe}_{3} \mathrm{O}_{4}$ ODE sample, $\sim 4.6 \times 10^{-13} \mathrm{erg}$ for $\mathrm{Ag} / \mathrm{Fe}_{3} \mathrm{O}_{4}$ PHE sample, and $\sim 8 \times 10^{-14} \mathrm{erg}$ for the $\mathrm{Fe}_{3} \mathrm{O}_{4}$ control sample. It is worth noting that the increase in $E_{\mathrm{bm}}$ also raises the anisotropy constant, whose mean value can be obtained from $K=E_{\mathrm{bm}} / V$, being $V$ the mean particle volume. Using this expression and assuming a mean diameter of $8.6 \mathrm{~nm}$ for the $\mathrm{Fe}_{3} \mathrm{O}_{4}$ nanocrystals, the $K$ values are $9.0 \times 10^{5} \mathrm{erg} / \mathrm{cm}^{3}$ and $1.4 \times 10^{6} \mathrm{erg} / \mathrm{cm}^{3}$ for dimer and flower-like samples, respectively. These values are appr. 3.5 and appr. 6 times larger than the obtained for the $\mathrm{Fe}_{3} \mathrm{O}_{4}$ control sample $(K$ from fit procedure is $\left.2.4 \times 10^{5} \mathrm{erg} / \mathrm{cm}^{3}\right)$. All the obtained values are/summarized in Table 1. Larger values of $E_{\mathrm{bm}}$ and $K$ can be partially attributed to an additional superficial disorder at the $\mathrm{Ag} / \mathrm{Fe}$-oxide interface. In that interface, magnetic moments could be isotropically pinned and still coupled with the magnetic moments of the magnetite core, generating a magnetic drag over the core that leads to the increase of the anisotropy [37]. Consequently, the coupling effects will be more significant for the sample with a greater interface between the two components, $\mathrm{Ag} / \mathrm{Fe}_{3} \mathrm{O}_{4} \mathrm{PHE}$ in this case. 

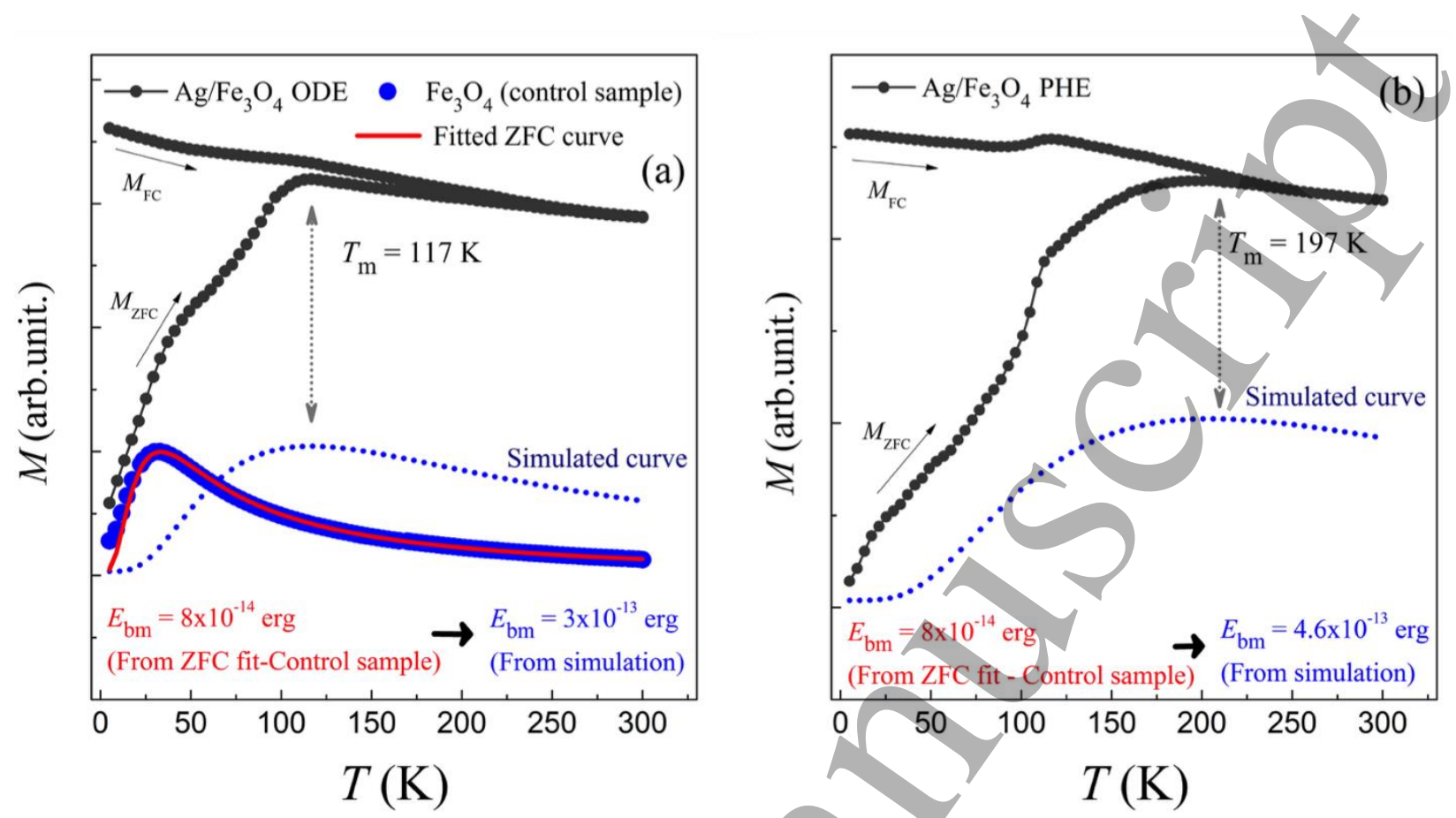

Figure 7. ZFC/FC curves taken in a magnetic field of 50 Oe for samples (a) $\mathrm{Ag} / \mathrm{Fe}_{3} \mathrm{O}_{4} \mathrm{ODE}_{\text {and }}$ (b) $\mathrm{Ag} / \mathrm{Fe}_{3} \mathrm{O}_{4}$ PHE. Blue filled symbols in (a) indicate the experimental ZFC curve of the magnetite control sample and the red solid line its respective fit according to Eq. 1. Blue dotted lines denote the simulated curves for the heterostructures according to Eq. 1.

Notice that it is not enough to just increase the value of the barrier energy (or $K$ ) to reproduce exactly the shape and the temperature evolution of the experimental ZFC curves obtained from the dual $\mathrm{Ag} / \mathrm{Fe}_{3} \mathrm{O}_{4}$ heterostructures. In order to achieve this goal for these complex structures, several other factors need to be considered, such as the dipolar interactions, possible effects of spin or charge transfer $[38,39]$ and cation exchange effects [40], structural defects [41] among others. 
Table 1. Parameters $E_{\mathrm{bm}}, T_{\mathrm{bm}}, K$ and $T_{i}$ obtained from the ZFC fit $\left(\mathrm{Fe}_{3} \mathrm{O}_{4}\right)$ and from simulations $\left(\mathrm{Ag} / \mathrm{Fe}_{3} \mathrm{O}_{4}\right.$ heterostructures).

\begin{tabular}{|c|c|c|c|c|}
\hline Sample & $E_{\mathrm{bm}}(\mathrm{erg})$ & $T_{\mathrm{bm}}(\mathrm{K})$ & $K\left(\mathrm{erg} / \mathrm{cm}^{3}\right)$ & $T_{\mathrm{i}}(\mathrm{K})$ \\
\hline $\begin{array}{c}\mathrm{Fe}_{3} \mathrm{O}_{4} \text { (Control } \\
\text { sample) }\end{array}$ & $8.0 \times 10^{-14(i)}$ & $21^{(i)}$ & $2.4 \times 10$ & \\
\hline $\begin{array}{c}\mathrm{Ag} / \mathrm{Fe}_{3} \mathrm{O}_{4} \mathrm{ODE} \\
\text { (Dimer-like } \\
\text { morphology) }\end{array}$ & $3.0 \times 10^{-13(i i)}$ & $73^{(i i)}$ & & 170 \\
\hline $\begin{array}{c}\mathrm{Ag} / \mathrm{Fe}_{3} \mathrm{O}_{4} \mathrm{PHE} \\
\text { (Flower-like } \\
\text { morphology) }\end{array}$ & $4.6 \times 10^{-13(i i)}$ & $120^{(i i)}$ & & 200 \\
\hline
\end{tabular}

Relevant information can also be qualitatively extracted from the FC magnetization curves. For example, the flattened behavior at low temperatures $(T \leq 50 \mathrm{~K})$ followed by a wider shoulder as the temperature increases could be a signature of limited nanoparticle aggregation and occurrence of a spin-glass and/or interacting superparamagnetic behavior [29, 42, 43]. Moreover, the manifestation of an irreversibility temperature $\left(T_{\mathrm{i}}\right)$, defined as the point in which the ZFC curve bifurcates from the FC one, indicates the presence of a distribution of blocking temperatures, as expected for size distributed magnetic monodomains. In summary, all examined properties suggest that the magnetic behavior of the studied samples are a consequence of a sum of several effects, leading to a complex interpretation of the magnetic results.

AC susceptibility measurements were performed to reinforce the results from DC magnetic characterizations. The real and imaginary components of the magnetic susceptibility vs. temperature ( $\chi$ 'vs. $T$ and $\chi^{\prime}$ vs. T), recorded at four different frequencies, are shown in Fig. 8. Although the $\chi^{\prime}(T)$ curves for $\mathrm{Ag} / \mathrm{Fe}_{3} \mathrm{O}_{4}$ nanoparticles follow different trends, two main features can be highlighted in both systems: an initial shoulder at low temperatures followed by a broader peak near the $T_{\mathrm{m}}$ value obtained from the ZFC measurements. The nature of these events can be related with two independent blocked-to-unblocked system processes. The equivalent two events in the imaginary component of the magnetic susceptibility $\chi{ }^{\prime \prime}(T)$ is reflected in the presence of 
two peaks, which are labeled as $T_{\operatorname{MAX}(1)}^{\chi^{\prime \prime}}$ and $T_{\operatorname{MAX}(2)}^{\chi^{\prime \prime}}$ in Fig. 8 (c and d). The initial shoulder at $\chi^{\prime}(T)$ is correlated to the first peak in $\chi^{\prime}(T)$ as expected, and can be assigned to the blocked-tounblocked transition of those iron oxide nanoparticles non-linked or weakly-linked with the Ag seeds. These temperature values are at similar positions to the ones observed in homogeneously nucleated nanoparticles (control $\mathrm{Fe}_{3} \mathrm{O}_{4}$ sample), as shown in Fig. S5 (Supporting Information). The second event, evidenced as a peak at $T \approx 110 \mathrm{~K}$ for the $\mathrm{Ag} / \mathrm{Fe}_{3} \mathrm{O}_{4} \mathrm{ODE}$ sample and $T \approx 170$ $\mathrm{K}$ for the $\mathrm{Ag} / \mathrm{Fe}_{3} \mathrm{O}_{4}$ PHE sample in the $\chi^{\prime}(T)$ and $\chi^{\prime \prime}(T)$ curves, is related to the unblocking processes of the $\mathrm{Fe}_{3} \mathrm{O}_{4}$ domains attached on the heterostructures or, in the case of flower-like sample with the unblocked-blocked transition of those magnetic nanoparticles with higher interparticle interaction. Notice that these peaks are registered at higher temperatures for the sample with flower-like morphology, an indication that in this sample the blocking/unblocking process requires more thermal energy to occur. This behavior also supports the previous evidence of the distinct interactions and possible alterations on the magnetocrystalline anisotropy between both systems.

The relative height of the peaks observed in the imaginary component of the magnetic susceptibility has significant differences, i.e., for the $\mathrm{Ag} / \mathrm{Fe}_{3} \mathrm{O}_{4} \mathrm{ODE}$ sample, $T_{\operatorname{MAX}(1)}^{\chi^{\prime \prime}}$ is higher $\operatorname{than} T_{\operatorname{MAX}(2)}^{\chi^{\prime \prime}}$, while for the $\mathrm{Ag} / \mathrm{Fe}_{3} \mathrm{O}_{4}$ PHE sample the opposite occurred. This difference can be related to the ratio of homogeneous to heterogeneous $\mathrm{Fe}_{3} \mathrm{O}_{4}$ domains, indicating that the reaction in phenyl ether avoids the homogeneous nucleation events and is more effective to produce heterostructures. 

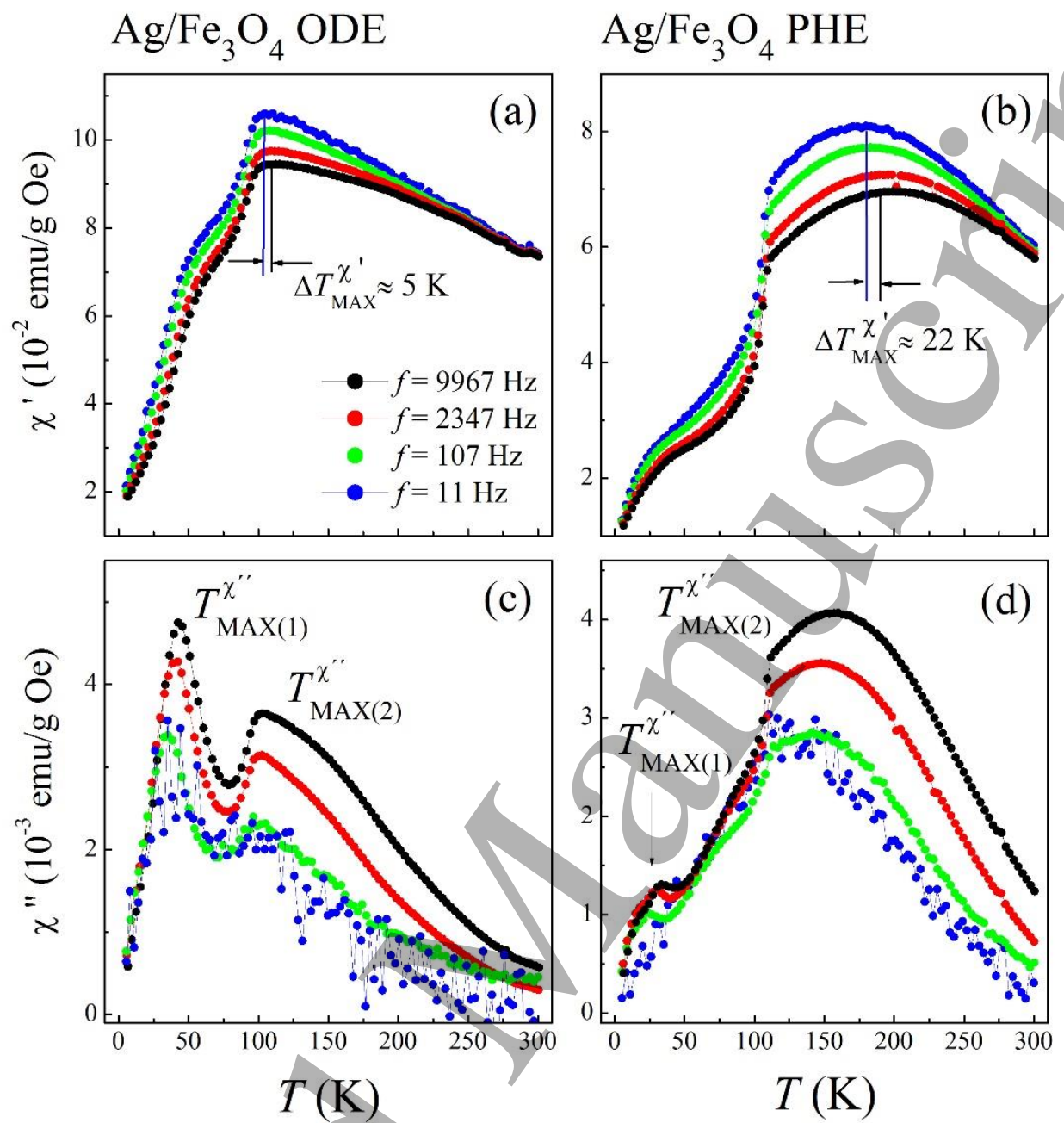

Figure 8. Temperature dependence of the real $\chi^{\prime}(T)$ and imaginary $\chi^{\prime \prime}(T)$ components of the susceptibility of the $\mathrm{Ag} / \mathrm{Fe} 3 \mathrm{O} 4$ heterostructures.

From the AC magnetization results, it is also possible to observe $T_{\mathrm{MAX}}$ displacements as frequency function. Such displacements are rather small when compared to the ones observed in highly interacting nanoparticle systems, a qualitative indication that the magnetic entities can be in a weakly interacting state [29, 31, 44, 45]. To clarify this point, the frequency dependence of the peaks in $\chi^{\prime}(T)$ was analyzed for both $\mathrm{Ag} / \mathrm{Fe}_{3} \mathrm{O}_{4}$ systems by fitting the experimental data using the Vogel-Fulcher law, given by:

$\tau=\tau_{0} \exp \left(E_{b} / k_{\mathrm{B}}\left(T-T_{0}\right)\right)$ 
where $\tau$ is the relaxation time that is associated to the frequency $f$ through $\tau=1 / f, \tau_{0}$ is the characteristic time (ranging between $10^{-9} \mathrm{~s}$ and $10^{-12} \mathrm{~s}[46]$ ), $k_{\mathrm{B}}$ is the Boltzmann constant, $E_{\mathrm{b}}$ is the energy barrier and $T_{0}$ is a characteristic temperature that defines if the thermal energy ( $T>$ $\left.T_{0}\right)$ or the interacting energy $\left(T<T_{0}\right)$ dominates the magnetic moment dynamics and magnetization process. By plotting the experimental data as $\ln (\tau)$ against $1 / T_{\mathrm{MAX}}^{\chi^{\prime}}$ (being $T_{\mathrm{MAX}}^{\chi^{\prime}}$ the peak associated to the blocking-unblocking processes of heterogeneous nucleated $\mathrm{Fe}_{3} \mathrm{O}_{4}$ domains) and using Eq. 1 to fit them (Fig. 9), parameters with coherent physical meaning were obtained. For $E_{\mathrm{b}}$, the determined values are $8 \times 10^{-14} \mathrm{erg}$ for $\mathrm{Ag} / \mathrm{Fe}_{3} \mathrm{O}_{4} \mathrm{ODE}$ and $2 \times 10^{-13} \mathrm{erg}$ for $\mathrm{Ag} / \mathrm{Fe}_{3} \mathrm{O}_{4} \mathrm{PHE}$, which are closer to the ones extracted from the analysís of the ZFC curves. Moreover, the obtained characteristic time $\tau_{0}\left(\sim 10^{-11}\right.$ and $\sim 10^{-10} \mathrm{~s}$ for the $\mathrm{Ag} / \mathrm{Fe}_{3} \mathrm{O}_{4}$ ODE and $\mathrm{Ag} / \mathrm{Fe}_{3} \mathrm{O}_{4}$ PHE samples, respectively) matches very well with those obtained from nanoparticle systems with moderate or weak interacting magnetic nanoparticles [46]. The larger values of $T_{0}$ determined for the sample with flower-like morphology also indicate a strengthening of the dipole-dipole and exchange forces due to intraparticle interactions. Using the Vogel-Fulcher law to analyze $\tau_{0}, E_{\mathrm{b}}$ and $T_{0}$ of those magnetic particles attached to the Ag seeds supports the previous conclusions obtained from DC magnetic studies, where larger energy barrier values were detected for linked magnetic nanoparticles. A summary of the extracted magnetic parameters is presented in Table 2. 


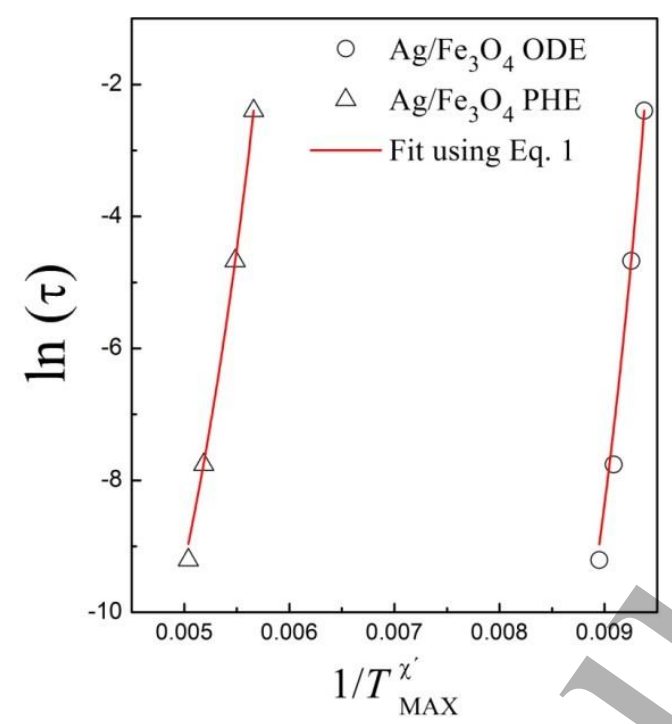

Figure 9. Plots of $\ln (\tau)$ vs. $1 / T_{\mathrm{MAX}}^{\chi^{\prime}}$ and the respective fit according to Eq. 1 (red solid line). Here, $T_{\text {MAX }}^{\chi^{\prime}}$ is the broader peak of the real component for every measured frequency.

Table 2. Extracted and calculated parameters from the AC magnetic susceptibility measurements.

30

3

\section{Conclusions}

Controlled synthesis protocols were employed to obtain magnetic-plasmonic nanoheterostructures of $\mathrm{Ag} / \mathrm{Fe}_{3} \mathrm{O}_{4}$ with two distinct morphologies. By controlling the polarity of the reaction medium, the charge compensation can be tuned, leading to particular architecture 
Acknowledgements

formations with $\mathrm{Ag}$ and iron oxide nanoparticles, resulting in dimer and flower-like configurations. Despite the fact that the magnetite nanoparticles in the heterocrystals have similar sizes and shapes, the magnetization response seems to reflect different phenomena. The observed dissimilarities on the AC and DC magnetization responses can be related to several factors. In the flower-like sample, the dipolar interactions between the Fe-oxide nanoparticles are stronger than in dimer-like counterpart. Also, in flower-like configuration one could have exchange interactions among Fe-atoms of neighboring particles, owing to the existence of percolated regions. Comparing the magnetic parameters of both $\mathrm{Ag} / \mathrm{Fe}_{3} \mathrm{O}_{4}$ samples with the $\mathrm{Fe}_{3} \mathrm{O}_{4}$ control sample and performed simulations, we detected important modifications in the magnetic response. The maximum of the zero-field-cooling curve was shifted around $90 \mathrm{~K}$ and $170 \mathrm{~K}$ for dimer and flower-like samples, respectively. Furthermore, the inferred magnetic anisotropies also reveal important variations. While part of these changes can be understood in terms of the strengthening of dipolar interactions or variations in the iron oxide phases, we also addressed the observed magnetic complex behavior from the coupling efficiency between metal/Fe-oxide and its role on the superficial disorder and hence on the magnetization response.

O. M.-L and M. K. acknowledge FAPESP and CNPq Brazilian agencies (2014/26672-8). The authors from Argentina thank CONICET. Brazilian Synchrotron Light Laboratory (LNLS/CNPEM) is acknowledged for XAS measurements (XAFS-17726). Brazilian Nanotechnology National Laboratory (LNNano/CNPEM) is acknowledged for the use of TEM JEOL JEM 2100 (21789) facilities. O.M.-L also thanks the Laboratory of Materials of the Autonomous University of Manizales (Colombia). IFW-Dresden and LNNano/CNPEM are acknowledged for TEM images. The authors thank Sebastian Calderon Velasco (INL, International Iberian Nanotechnology Laboratory) for the valuable contributions to the discussion. 
References

[1] Melinon P, Begin-Colin S, Duvail J L, Gauffre F, Boime N H, Ledoux G, Plain J, Reiss P, Silly F and Warot-Fonrose B, 2014 Phys. Rep. 543 163-197.

[2] Xia Y, Gilroy K D, Peng H C and Xia X 2017 Angew. Chem. 56 60-95.

[3]. Veverka M, Zaveta K, Kaman O, Veverka P, KnIzek K, Pollert E, Burian M and Kaspa P 2014 J. Phys. D: Appl. Phys. 47065503

[5] Costa L S and Zanchet D 2017 Catal. Today. 282 151-158.

[5] Wang C, Yin H, Dai S and Sun S 2010 Chem. Mater. 22 3277-3282.

[6] Amarjargal A, Tijing L D, Im I T and Kim C S 2013 Chem. Eng. J. 226 243-254.

[7] Gong P, Li H, He X, Wang K, Hu J, Tan W, Zhang S and Yang X 2007 Nanotechnology. 18 285604.

[8] Brollo M E F, Orozco-Henao J M, López-Ruiz R, Muraca D, Dias C S B, Pirota K R and Knobel M 2016 J. Magn. Magn. Mater. 397 20-27.

[9] Das R, Rinaldi-Montes N, Alonso J, Amghouz Z, Garaio E, García J A, Gorria P, Blanco J A, Phan M H and Srikanth H 2016 ACS Appl. Mater. Interfaces. 8 25162-25169.

[10] Sun L, He J, An S, Zhang J and Ren D 2013 J. Mol. Struct. 1046 74-81.

[11] Yu H, Chen M, Rice P M, Wang S X, White R L, Sun S 2005 Nano. Lett. 2 379-382.

[12] Moscoso-Londoño O, Muraca D, Tancredi P, Cosio-Castañeda C, Pirota K R, Socolovsky L M, 2014 J. Phys. Chem. C. 118 13168-13176.

[13] Y. Sun 2015 Natl. Sci. Rev. 2 329-348.

[14] Brollo M E, Lopez-Ruiz R, Muraca D, Figueroa S J, Pirota K R and Knobel M 2014 Sci. Rep. 46839.

[15] Chandra S, Huls N A, Phan M H, Srinath S, Garcia M A, Lee Y, Wang C, Sun S, Iglesias O and Srikanth H 2014 Nanotechnology. 25055702.

[16] Choi S H, Na H B, Park Y I, An K, Kwon S G, Jang Y, Park M H, Moon J, Son J S, Song I C, Moon W K and Hyeon T, S 2008 J. Am. Chem. Soc. 130 15573-15580.

[17] Hyeon T, Lee S S, Park J, Chung Y and Na H B 2001 J. Am. Chem. Soc. 123 12798-12801. [18] Vreeland E C, Watt J, Schober G B, Hance B G, Austin M J, Price A D, Fellows D B, Monson T C, Hudak N S, Maldonado-Camargo L, Bohorquez A C, Rinaldi C and Huber D L 2015 Chem. Mater. 27 6059-6066. 
[19] Wang C, Xu C, Zeng H and Sun S 2009 Adv. Mater. 21 3045-3052.

[20] Huang J, Sun Y, Huang S, Yu K, Zhao Q, Peng F, Yu H, Wang H and Yang J, 2011 J. Mater. Chem. 2117930.

[21] Wei Y, Klajn R, Pinchuk A O, Grzybowski B A 2008 Small. 4 1635-1639.

[22] Gallo J, García I, Padro D, Arnáiza B and Penadés S 20102011 J. Mater. Chem. 20 1001010020.

[23] Hao D, Cheng-Min S, Chao H, Zhi-Chuan X, Chen L, Yuan T, Xue-Zhao S and Hong-Jun G 2010 Chin. Phys. B. 19066102.

[24] Zhang L, Dou Y H and Gu H C 2006 J. Colloid. Interface. Sci. 297 660-664.

[25] Fantechi E, Roca A G, Sepúlveda B, Torruella P, Estradé S, Peiró F, Coy E, Jurga S, Bastús N G, Nogués J and Puntes V 2017 Chem. Mater. 29 4022-4035.

[26] Schladt T D, Graf T, Köhler O, Bauer H, Dietzsch M, Mertins J, Branscheid R, Kolb U and Tremel W 2012 Chem. Mater. 24 525-535.

[27] Muraca D, Sharma S K, Socolovsky L M, de Siervo A, Lopes G and Pirota K R 2012 J. Nanosci. Nanotechnol. 12 6961-6967.

[28] Knobel M, Nunes W C, Socolovsky L M, Biasi E D, Vargas J M and Denardin J C 2008, J. Nanosci. Nanotechnol. 8 2836-2857.

[29] Socolovsky L M and Moscoso-Londoño O. 2017 Consequences of Magnetic Interactions Phenomena in Granular Systems, in: S.K. Sharma (Ed.) Complex Magnetic Nanostructures Synthesis, Assembly and Applications, Springer.

[30] Orozco-Henao J M, Coral D F, Muraca D, Moscoso-Londoño O, Mendoza Zélis P, Fernandez van Raap M B, Sharma S K, Pirota K R and Knobel M 2016 J. Phys. Chem. C. 120 12796-12809.

[31] Moscoso-Londoño O, Tancredi P, Muraca D, Mendoza Zélis P, Coral D, Fernández van Raap M B, Wolff U, Neu V, Damm C, de Oliveira C L P, Pirota K R, Knobel K R and Socolovsky L M/2017 J. Magn. Magn. Mater. 428 105-118.

[32] Tancredi P, Rivas Rojas P C, Moscoso-Londoño O, Wolff U, Neu V, Damm C, Rellinghaus B, Knobel M and Socolovsky L M 2017 New J. Chem. 41 15033-15041.

[33] Jiang J, Gu H, Shao H, Devlin E, Papaefthymiou G C and Ying J Y 2008 Adv. Mater. 20 4403-4407. 
[34] Umut E, Pineider F, Arosio P, Sangregorio C, Corti M, Tabak F, Lascialfari A and Ghigna P 2012 J. Magn. Magn. Mater. 324 2373-2379.

[35] Muraca D, de Siervo A and Pirota K R 2012 J. Nanopart. Res. 151375

[36] Hansen M F and Mørup S 1999 J. Magn. Magn. Mater. 203 214-216.

[37] Rondinone A J, Liu C and Zhang Z J 2001 J. Phys. Chem. B. 105 7967-7971.

[38] Pineider F, Fernández C D J, Videtta V, Carlino E, Hourani A A, Wilhelm F, Rogalev A, Cozzoli P D, Ghigna P and Sangregorio C 2013 ACS Nano. 7 857-866.

[39] Schick I, Gehrig D, Montigny M, Balke B, Panthöfer M, Henkel A, Laquai F and Tremel W 2015 Chem. Mater. 27 4877-4884.

[40] Sytnyk M, Kirchschlager R, Bodnarchuk M I, Primetzhofer D, Kriegner D, Enser H, Stangl

J, Bauer P, Voith M, Hassel A W, Krumeich F, Ludwig F, Meingast A, Kothleitner G, Kovalenko M V and Heiss W 2013 Nano. Lett. 13 586-593.

[41] Laha S S, Regmi R and Lawes G 2013 J. Phys. D: Appl. Phys. 46325004.

[42] León Félix L, Coaquira J A H, Martínez M A R, Goya G F, Mantilla J, Sousa M H, Valladares L D L S, Barnes C H W, Morais P C 2017 Sci. Rep. 741732.

[43] Morales M B, Phana M H, Pal-S, Frey N A and Srikantha H 2009 J. Appl. Phys., 105 $07 \mathrm{~B} 511$.

[44] Masunaga S H, Jardim R F, Fichtner P F P and Rivas J 2009 Phys. Rev. B. 80184428.

[45] Landi G T, Arantes F R, Cornejo D R, Bakuzis A F, Andreu I, Natividad E, J. Magn. Magn. Mater. 421 138-151

[46] Lee J S, Tan R P, Wu J H and Kim Y K 2011 Appl. Phys. Lett. 99062506. 